\title{
Apparel Fit Assessment Using Parametric Models
}

\author{
Pinkie E. ZWANE* \\ University of Swaziland (UNISWA), Textiles, Apparel Design \& Management, Luyengo, Swaziland \\ DOI: $10.15221 / 15.029$ http://dx.doi.org/10.15221/15.029
}

\section{Introduction}

Apparel fit is a concern not only to apparel manufacturers who endeavour to produce better fitting ready-to-wear apparel, but it is also crucial to apparel retailers, who strive to sell satisfactory apparel products to consumers, that fit well. Better fitting apparel will help increase sales and reduce returns of ill-fitting garments. Apparel fit is how well a garment conforms to the 3D of the human body (Brown and Rice, 2001). It mostly entailed appearance and comfort assessments that are highly subjective and led to the satisfaction of consumers with an apparel in a still position. In functional clothing, apparel fit also factors the ability to accommodate movement of the body (Boorady, 2011). With most fashionable garments, fit can be categorized into three: tailored fit which is described as perfect fit, body con (tight) fit aimed at showing off the wearer's curves and oversize fit, for intentionally loose garments (Cox, 2012).

For the above described fit to be achieved, it corresponds to the amount of ease included during the pattern making and construction of the garment. For tailored fit, wearing ease is included; for body hugging or tight fitting garments, wearing and design ease are excluded, and for the oversize fit, design ease is included. Most conducted studies on garment fit are mainly on the tailored fit, where the apparel skims the contours of the body and is eased in all the right places to achieve a perfect fit (Cox, 2012).

Given the subjective nature of fit assessment, where expert panellists have been used to generate good data, attempts of objective assessments have been introduced through virtual fitting applications with colour indicators to show tight fitting areas of the garment that need alteration. Garment simulation enables designers, pattern makers and apparel manufacturers to present style decisions, test the fit of a garment in less time than actual sewing and share the results instantly, without expensive sewing and shipping costs (Olaru, et al., 2012). Virtual apparel fitting allows consumers to visualise how garments look like when worn without physically wearing them (Zhou et al., undated). Many pattern makers use 3D garment simulation to test their pattern blocks and while drafting the pattern, to ensure that the drape and general fit of the garment are correct (Olaru, et al., 2012).

Virtual fitting has also paved a way for increased e-shopping, a retailing option that has gained popularity in recent years. The available computer aided design and manufacturing software packages have different capabilities. Hence the use of the garment simulation software will be discussed in the pilot project done in South Africa. The purpose of the study was to explore the capability of the Optitex software package in assessing fit on parametric models. The pilot project was guided by the following objectives: to determine the ease of using the software package in assessing fit virtually, and to assess fit of simple styled skirts using different fabrics for the benefit of the apparel manufacturing and retailing sectors.

\section{Methods}

The project utilized an experimental approach. The optitex software was procured from the supplier overseas in order to explore if it could address the challenge of ill-fitting garments given the unavailability of updated anthropometric data, prevalent body shapes in Southern Africa and to enhance e-commerce efforts in the apparel sector. Two fabric types were purposively selected from the fabric gallery provided by the software. One was floral light-weight with good draping properties, the other one was a plain colour medium-weight fabric.

\footnotetext{
*Tel: (268) 25170520 (O), Fax: (268) 25274441, E-mail: pezwane@uniswa.sz
} 
Parametric models were used to fit styled skirts made from the two fabric types. The body measurements of the parametric models were adjusted on the lower torso to two body shapes prevalent in Southern Africa, which are the triangular and hourglass shapes. The body shapes were identified using body measurements from the $\mathrm{TC}^{2}$ scanner and drop values were used to determine the body shapes. The garment assembly simulation was performed using imported Gerber AccuMark patterns of a classic straight skirt and A-line skirt silhouettes. Adjusted parametric models were then used to assess the fit on them using the tension map tool of the Optitex software. The tension map has 3 different colours: blue if the garment is loose, green if the garment is perfect fit, red if the garment is too tight (Olaru, et al., 2012).

\section{Results and Discussion}

Operating the software was relatively easy although the technical assistance was not available locally. Training in the use of the software was offered once when the software was installed, yet the proficiency in using the software needed enhancement to improve competency. The hourglass shaped model was fitted the classic skirt in a plain coloured fabric. A tight fit resulted along the hip line, indicated by an orange to red colour. Figure 1 shows fitted classic skirt without the activated tension map tool. On the illustration below, one can see the tight fit along the hip area. Ideally the orange to red colour should show as seen in Figure 2 from Wu, et al., (2011), indicating the tight fit in hip line area.

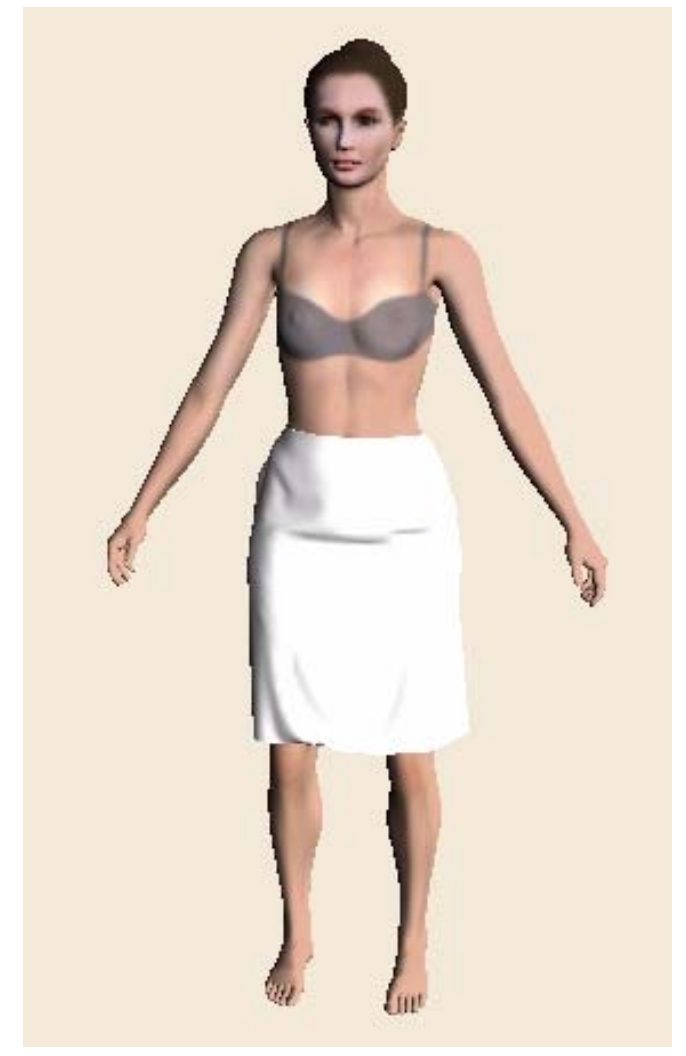

Figure1. Assessment of a classic skirt on an hourglass shaped model

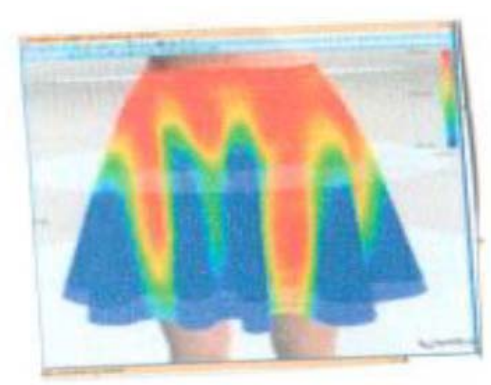

Figure 2. Colour indicators of the tension map tool 
The orange to red colour on Figure 1 was on the hip area, although not seen on the figure. That indicated the hip line area was tight fitting and would require an adjustment in the pattern size around the hipline to achieve a tailored fit of the skirt. After adjusting the patterns, the orange colour then turned yellow to green for a proper fit. The A-line floral skirt was tried on the hourglass as shown in Figure 3. Black and white illustrations of the A-line skirt on the hour body shape showed a slightly perfect to loose fit shown by a yellow to green colour indicator.

The medium weight fabric was not tried on the triangular body shape because Rasband (2002); Marshall, Jackson and Stanley (2012) recommend flared skirts on the triangular body shape to accentuate the body shape profile. Hence, the floral light weight fabric was tried on the triangular body shape. The results shown in Figure 3 indicate that the skirt had a slight loose fit on the triangular body shape profile particularly on the waist from the way the garment drapes over the model. When the tension map tool was used, the colours were yellow to blue showing that the skirt was not close to the body. The tension map tool or software system operated in a similar way to the infra-red light. When the light is focused on a living thing, it picks up the heat energy from the body and indicates its location by a red light. With the tension map tool, the closer the fabric to the body the more red it became.

Having used the software in this pilot project showed that if body scans were imported from the $\mathrm{TC}^{2}$ scanner data file, a better fit on virtual models could have resulted because the body contours of the parametric models were not similar to the body scans from the scanner, despite having used the same body measurements from the scanner for adjusting the parametric models. This assertion was also noted by Protopsaltou et al. (2002), who were going to explore the use of human virtual models instead of the parametric models in order to get better fit assessments. Although average measurements for determining the body shapes were used, the shape was still influenced the drape of fabric on the model. A seamless functioning of the scanner and Optitex software could have enhanced the results on the virtual fitting of the skirts. Compatibility of software packages could benefit the users and enhance the use of the garment simulation software systems by apparel manufacturers and retailers because some companies already have other versions of the computer aided design software packages.
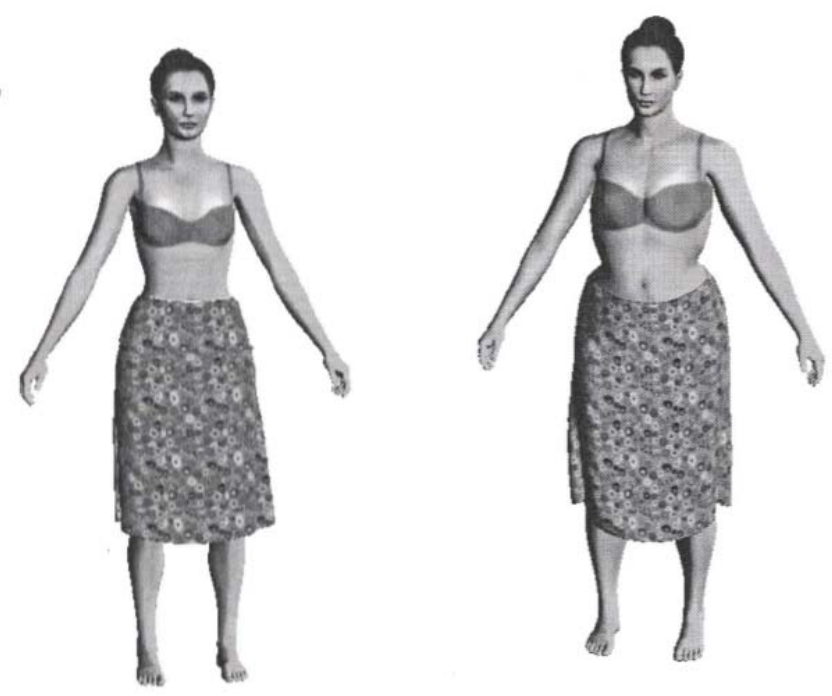

Figure 3. Assessment of an A-line skirt on an hourglass and triangular shaped models

From the findings, a proposed conceptual model in assessing fit using the Optitex Runway software is presented in Figure 4. Body measurements would be extracted from the scanned data and fed into the computer-aided design software for pattern generation. After pattern making, fabric characteristics would be selected and used for the garment assembly to take place using the selected garment style. Fit would be assessed on the scanner body models using the tension map tool to show through the colour indicators how the garment fits the model. The use of virtual human models has been noted by researchers in MIRALab, that using virtual human add value in fit assessments (Protopsaltou et al., 2002). 


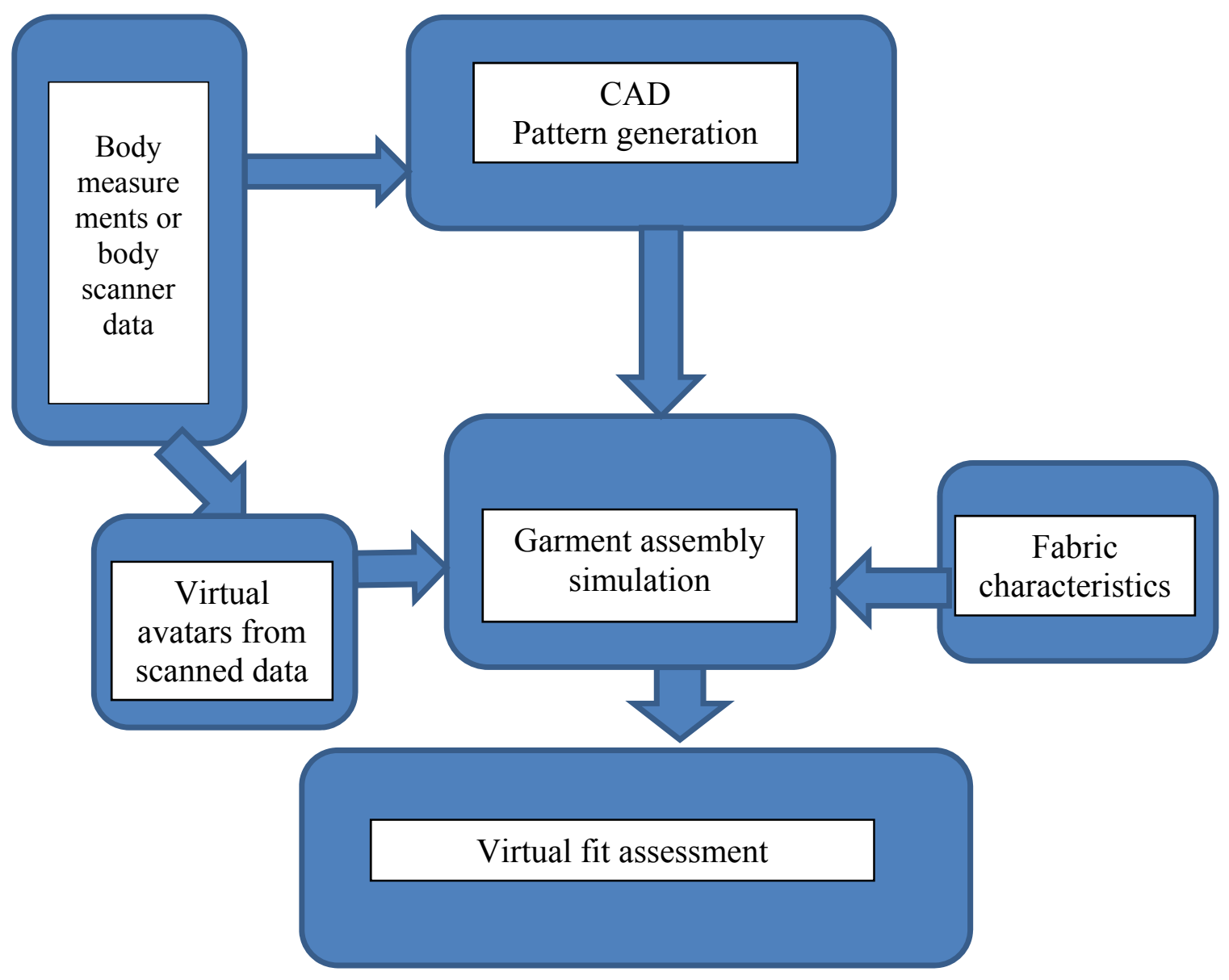

Figure 4. Conceptual model for assessing fit virtually

\section{Conclusion}

The software is definitely useful in the apparel manufacturing industry. Pattern making is cut by almost half to three-quarters and the construction time is drastically reduced. Fit trials are also drastically reduced. There are realistic benefits to the garment industry in developed countries (Maja and Slavenka, 2013). Benefits of apparel design and garment simulation systems for improved fit include the following for retailers: increased sales, more products sold at undiscounted prices, increased customer satisfaction and retention, reduced garment returns and customer loyalty and growth. For manufacturers, the benefits are more accurate product development input data, reduced sample making budgets, shortened time to market, better fitting garments, and improved quality control (Yu, 2009).

Simulations of skirts in assessing fit using the Optitex 3D runway software proved to be valuable. The Optitex Runway software is very good at visualizing the fit and drape of apparel on the parametric models. The software tool enabled e-prototyping to be realised without incurring the actual production cost of a real sample. The tension map tool assisted in identifying areas that needed more ease on the garment to achieve better fit.

It would add more value if body scanner avatars were to be used for better and effective fit results. The same body measurements of a model give different cross sectional views of the lower torso, body shapes and postures which influence the hang of the garment on the model. Fit assessment on the parametric models assisted in visually detecting areas where pattern adjustments were needed. The classic skirt was tight fitting on the hourglass shaped parametric model, yet the A-line fitted well. The flared skirt on the triangular shaped model had a slightly loose fit especially on the waist line. The fit on the waist line underscores the importance of using the human avatars from the scanner for better fitting assessments to be made. 
Valuable information is obtained on ill-fitting garments using the parametric models, but the challenge is the absence of an expert system to estimate the percentage or extent for adjusting the original patterns from the indicated colour for better fitting garments. Such a system would fast track the pattern making and fitting processes instead of the trial and error that was done using the parametric models. Similar limitations with parametric models in addressing fit issues were also expressed by Maja and Slavenka (2013) who felt there was no single rule to use in adjusting the computer based parametric garment models for the men's jacket that was tested for fit. They felt it was necessary to analyse each pattern separately for proper adjustment to be made.

The proposed model can assist in improving the current simulation software packages for better garment fit simulations. Furthermore, it will identify areas of interface that can improve the functionality of the Optitex sotware.

\section{References}

Boorady, L.M. (2011). Functional clothing - Principles of fit. Indian Journal of Fibre and Textile Research, 36, 344-347.

Brown, P. and Rice, J. (2001). Ready-to-wear apparel analysis. Prentice Hall Inc., New Jersey.

Cox, A. (2012). The three different definitions of correct clothing fit. http://youlookfab.com/2012/09/20/the-three-different-definitions-of-correct-clothing-fit/. 21/08/15.

Maja, M. and Slavenka, P. (2013). Investigation of fit of computer based parametric garment prototypes.

Marshall, S.G., Jackson, H.O. and Stanley, S.M. (2012). Individuality in clothing selection and personal appearance $\left(7^{\text {th }}\right.$ Edition). Prentice Hall, New York.

Olaru, S., Filipescu, E., Filipescu, E., Niculescu, C., \& Salistean, A. (2012). 3D fit garment simulation based on $3 d$ body scanner anthropometric data. Proceedings of the 8th International DAAAM Baltic Conference titled "Industrial Engineering"held on the 19-21 April at Tallinn, Estonia.

Protopsaltou, D., Luible, C., Arevalo, M. \& Thalmann, N. (2002). A body and garment creation method for an internet based virtual fitting room. http://link.springer.com/chapter/10.1007\%2F978-14471-0103-1 7. 27/08/2015.

Rasband, J. (2002). Wardrobe strategies for women. Fairchild, New York.

$\mathrm{Yu}, \mathrm{W}$. (2009). Current and future industrial applications of 3D body scanners. Paper presented at the Seminar held at the University of Pretoria, South Africa, on the $9^{\text {th }}$ February 2009.

Zhou, Z., Shu, B., Zhuo, S., Deng, X., Tan, P. and Lin, S. (undated). Image-based clothes animation for virtual fitting. http://research.microsoft.com/enus/um/people/stevelin/papers/sigasiabrief12.pdf. 28/05/2015. 